\title{
REGRESSION MODEL FOR ANALYZING THE DGS STRUCTURES PROPAGATION CHARACTERISITCS
}

\author{
Prakash K Kuravatti ${ }^{1}$, T. S. Rukmini ${ }^{2}$ \\ ${ }^{I}$ Asst Professor, EEE Dept. Alliance College of Engineering \& Design, Bangalore India \\ ${ }^{2}$ Professor, ECE Dept., Nitte minakshi Institute of technology, Bangalore, India
}

\begin{abstract}
Defected ground structure (DGS) analyzed by recently due to the great potential capability of microwave application. The propagation characteristic of DGS is varied based on their structure changes and it affects the performance of designed microwave antenna. For analyzing the propagation characteristics in a clear way, there is a need for modeling of the structure. This mathematical model determines the performance of DGS structures and realizes the slow wave effect. Using the proper DGS structure, there is a possibility of good slow wave effect and Q-factor. Moreover, the surface wave polarization of DGS structure is also affected by the microwave integrated circuit. In the paper, regression analysis based mathematical model is proposed to analyze the propagation characteristics of DGS. Regression models are one of the statistical models which describe the variation in one or more variables when one or more other parameters vary. The proposed regression based mathematical model is implemented in MATLAB working platform and the output performance is evaluated with Rectangular DGS structures such as The effectiveness of the proposed regression model is validated by evaluating error deviations of it with curve fitting model and experimental model for mentioned PDGS structures.
\end{abstract}

Keywords: DGS, Propagation Characteristics, Experimental Model, Mathematical Model, Curve Fitting, Uncertainty, regression model

\section{INTRODUCTION}

Currently, the defected ground structure (DGS) is gaining much attention in the fields of microwave and millimeter wave applications [1]. It is extensively used in microwave circuit design such as power divider, power amplifier and specifically in filter design [3] High performance, compact size and low expense often meets the stringent requirements of modern microwave communication systems [4]. Ultra-wideband (UWB) technology has turned into one of the most competent solutions for future short-range high-speed indoor data communication applications [9]. Nowadays, there is an increasing interest in developing UWB radio for short-range high-speed wireless communication networks [10]. DGS is realized by engraving off a simple shape defect from the ground plane. The shielded current distribution in the ground plane is disturbed based on the shape and dimensions of the defect, resulting in a controlled excitation and propagation of the electromagnetic waves via the substrate layer [5]. The effect of DGSs is to place parallel inductance and capacitance in the lumped model, which includes a pole to the transfer function and results in a resonant frequency [7]. DGS has the characteristics of stop-band, slow-wave effect, and high impedance that have been created to reduce the harmonics as well as to realize the compact physical dimensions of RF circuits [8].
Today, DGS for the micro strip lines that has engraved defects in the backside metallic ground plane has become one hot area in microwave integrated circuits (MIC) design [6]. In numerous wireless communication systems, micro strip patch antennas are utilized widely because of their several benefits such as low profile, light weight, easy fabrication and conformability to mounting structure [12]. EBG structures realized on metal layers are helpful for creating filters such as band-stop filters, low-pass filter and band-pass filter, phase shifters and antennas [15]. Numerous shapes of DGS slot have been studied in planar micro strip antenna designs, which provides better performances- size diminution (lower resonant frequency), impedance bandwidth enhancement (lower quality factor), and gain increasing [11]. DGS have received substantial interests, because they can refuse certain frequency bands, and hence it is called electromagnetic band gap (EBG) structures [2]. DGS and the Electromagnetic band gap (EBG) structures, generally called as the photonic band gap structures (PBG), are the two diverse types of generic structures employed for the design of the compact and high performance microwave components [13]. Application of DGS and EBG will minimize the dimensions of the divider as well as improves its scattering parameters [7]. The DGS reduce the mutual coupling between the microwave antenna array elements. The effect of mutual coupling increases the propagation uncertainty of microwave antenna. So, the accuracy of the propagation of microwave is affected and 
more chance for occurring confliction in the DGS size. To overcome this problem, regression based mathematical model is used for analyzing the experimental model uncertainty.

\section{PROBLEM STATEMENT AND PROPOSED METHODOLOGY}

A defected ground structure etched in the metallic ground plane of a micro strip line is attractive solution for achieving finite pass band, rejection band and slow-wave characteristics [21]. It has been widely used in the development of miniaturized antennas. Many DGS patterns such as dog bone structure, spiral DGS, H-, U- and T-shaped DGSs are presented in the literature for achieving high Q-factor [22]. From the related works in above section, the different DGSs and their propagation characteristics are analyzed. The propagation characteristics of the microwave applications depend on DGS dimensions and slot distance which improves the performance of the microwave antenna. Due to the varied application structure of DGS, there is a chance of propagation uncertainty. Hence, for analyzing the propagation characteristics of DGS it is necessary to build a mathematical model using the experimental data. With the proper mathematical model, it is possible to improve the performance, slow wave effect and Q-factor also. In this paper, a regression analysis based mathematical model is proposed for analyzing the propagation characteristics and its uncertainty of DGS structure. The proposed mathematical model is based on the various kinds of DGS and their microwave propagation characteristics. The different kinds of DGS, the equivalent circuit model, and the proposed methodology are described in the following sections.

\subsection{Different Model of DGS and Equivalent Circuit}

Defected Ground Structures (DGS) are implemented by etching slots of different shapes in the ground planes. They have been employed to improve the performance in multiple applications such as filters, couplers or dividers, microwave circuits as well as to reduce the mutual coupling between the elements of antenna arrays. In this application, the parameters considered are the shape of unit DGS, the distance between two DGS, and the distribution of different DGS. As well as the DGS can improve the performance of the antenna, restrain harmonious, and decrease the mutual coupling of antenna array by containing the surface waves. The additional equivalent components and the increased slow wave effect are the important properties of DGS because, it can be realized and the equivalent circuit can be reduced using these properties.

\subsection{Regression Analysis based Mathematical Model}

In existing works, there is no proper model for analyzing the uncertainty of wave dispersion and transmission characteristics of DGS structures. A model is needed to determine the performance of DGSs and realizes the slow wave effect. Here, the regression analysis is the process of developing a mathematical model. For developing the mathematical model, data of characteristics is generated from experimental results. From the developed model, the uncertainty of the propagation characteristics is analyzed. The experimental data is obtained from the propagation characteristics of Periodic Defected Ground Structure (PDGS). Then, the regressive model is chosen as the reference model and propagation uncertainty of the experimental data is determined with it.

The Rectangular DGS structure is chosen to develop the autoregressive mathematical model and to analyze the propagation uncertainty. The chosen Rectangular PDGS. Then the considered DGS structures dispersion and the surface power transmission of microwave are analyzed for propagation uncertainty. Using the considered characteristics, the regression process is applied to develop the mathematical model.

The steps used for developing the regression based mathematical model are described as follows.

Step 1: Initialize the DGS structure and the appropriate experimental microwave characteristics,

Step 2: Develop the general equation with propagation coefficients on $\mathrm{y}$-axis and frequency components on $\mathrm{x}$-axis,

Step 3: Apply regression process on the general equation and develop the mathematical model,

Step 4: Determine the uncertainty of the experimental characteristics.

The chosen general expression for regressive process to develop the mathematical model is provided as

$$
y_{n}=\frac{1}{1+\exp \left(-w_{0}-w_{1} x_{1}-w_{2} x_{2} \ldots-w_{n} x_{n}\right)}
$$

Using logarithms on both sides, the above equation can be rewritten as

$$
\ln \left(\frac{y_{n}}{1-y_{n}}\right)=-w_{0}-w_{1} x_{1}-w_{2} x_{2} \ldots-w_{n} x_{n}
$$

Where

$w_{0}, w_{1}, w_{2}, \ldots, w_{n}$ are coefficients of regression analysis. From the equation (4.b), the rectangular PDGS microwave dispersion characteristics and the surface wave characteristics are developed as illustrated below.

$$
\ln \left(\frac{\left(\beta / k_{0}\right)_{n}}{1-\left(\beta / k_{0}\right)_{n}}\right)=-w_{0}-w_{1} f_{1}-w_{2} f_{2} \ldots-w_{n} f_{n}
$$




$$
\ln \left(\frac{(S 21)_{n}}{1-(S 21)_{n}}\right)=-w_{0}-w_{1} f_{1}-w_{2} f_{2} \ldots-w_{n} f_{n}
$$

Where

$\beta / k_{0}, \quad S 21$ and $f$ are the microwave dispersion coefficient, transmission coefficient and frequency component in $\mathrm{kHz}$.

The matrix representations of the equation (5) and (6) for improving the regression based mathematical model are illustrated as

$$
\begin{aligned}
& {\left[\begin{array}{c}
\ln \left(\frac{\left(\beta / k_{0}\right)_{1}}{1-\left(\beta / k_{0}\right)_{1}}\right) \\
\ln \left(\frac{\left(\beta / k_{0}\right)_{2}}{1-\left(\beta / k_{0}\right)_{2}}\right) \\
: \\
\ln \left(\frac{\left(\beta / k_{0}\right)_{n}}{1-\left(\beta / k_{0}\right)_{n}}\right)
\end{array}\right]=\left[\begin{array}{ccccc}
1 & f_{1} & 0 & \ldots . . & 0 \\
1 & f_{1} & f_{2} & \ldots \ldots & 0 \\
: & : & : & \ddots & : \\
: & : & : & \ddots & : \\
1 & f_{1} & f_{2} & \ldots . . & f_{n}
\end{array}\right]\left[\begin{array}{c}
-w_{0} \\
-w_{1} \\
: \\
: \\
\vdots \\
-w_{n}
\end{array}\right]} \\
& {\left[\begin{array}{c}
\ln \left(\frac{(S 21)_{1}}{1-(S 21)_{1}}\right) \\
\ln \left(\frac{(S 21)_{2}}{1-(S 21)_{2}}\right) \\
: \\
\ln \left(\frac{(S 21)_{n}}{1-(S 21)_{n}}\right)
\end{array}\right]=\left[\begin{array}{ccccc}
1 & f_{1} & 0 & \ldots . . & 0 \\
1 & f_{1} & f_{2} & \ldots . . & 0 \\
: & : & : & \ddots & : \\
: & : & : & \ddots & : \\
1 & f_{1} & f_{2} & \ldots . . & f_{n}
\end{array}\right]\left[\begin{array}{c}
-w_{0} \\
-w_{1} \\
: \\
: \\
: \\
-w_{n}
\end{array}\right]}
\end{aligned}
$$

By solving equations (7) and (8), we can get the estimated values of coefficients $\left(w_{0}, w_{1}, w_{2}, \ldots, w_{n}\right)$ of regression analysis. Select the coefficients of regression model such the mean square error (MSE) is minimized to zero. After computing the coefficients of regression process, we can able to develop the mathematical model which represent the propagation coefficients. Then, the uncertainty propagation of the experimental characteristics are analyzed and compared with the proposed regression based mathematical model.

\section{RESULTS AND DISCUSSION}

The proposed regression analysis based mathematical model is implemented in MATLAB working platform. Based on the experimental results, regression based mathematical model is developed. Then, the propagation characteristics of developed mathematical model are determined for DGS structure. The analyzed DGS structures is rectangular. The considered propagation characteristics are dispersion characteristics and surface wave transmission characteristics. From the determined propagation characteristics of DGS structure, their propagation uncertainties are computed and error deviation of the proposed model with experimental model and curve fitting mathematical is evaluated for mentioned PDGS structures. The dispersion and surface transmission of microwave of rectangular PDGS are described as follows.

\subsection{Rectangular PDGS:}

The dispersion characteristics and the surface wave transmission characteristics of rectangular PDGS are shown in Fig.1 and Fig.2.
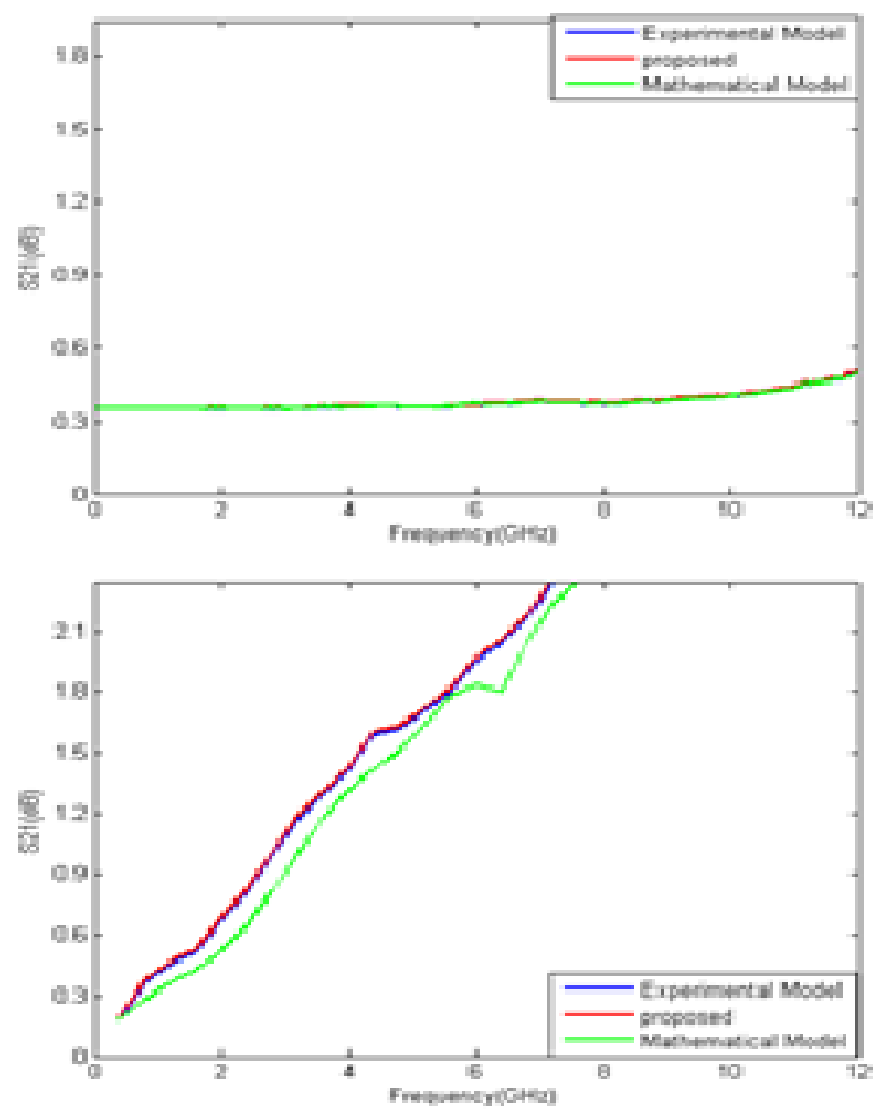

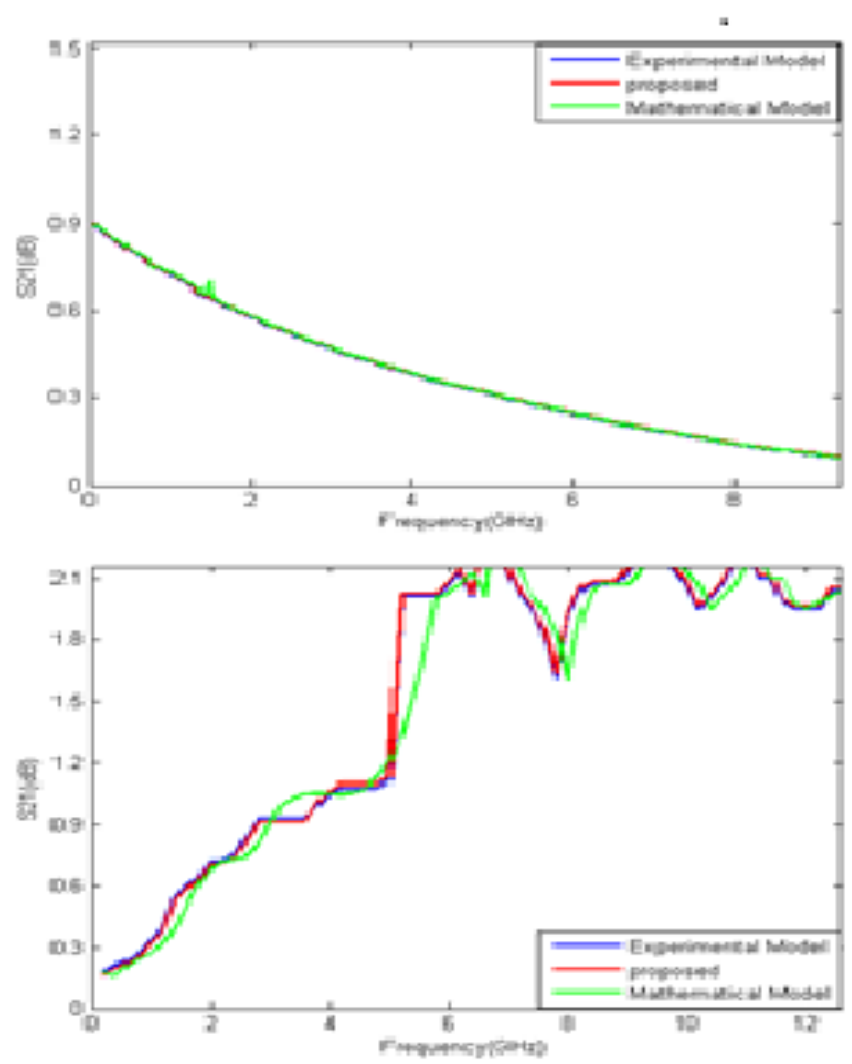

Fig 1: Dispersion Characteristics of Rectangular PDGS.

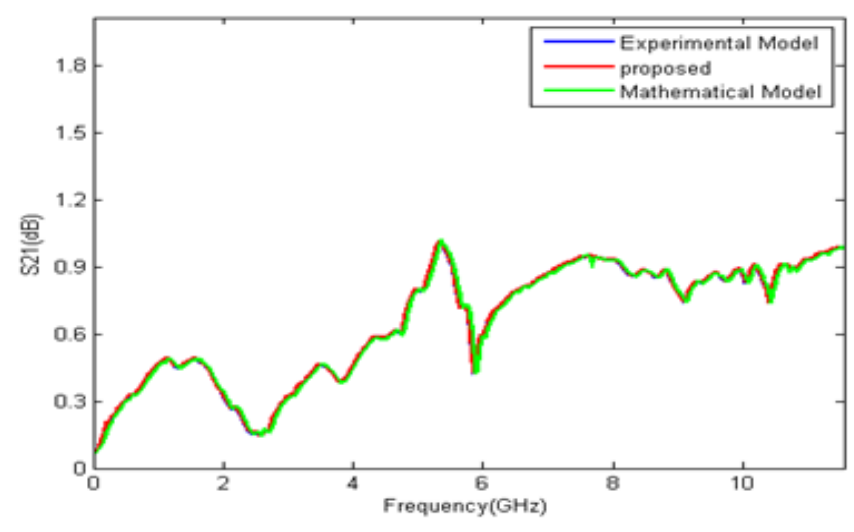

Fig 2: Surface wave Transmission Characteristic of Rectangular PDGS.

Here, in rectangular PDGS, cells are arranged as an array in the same spacing direction. At different periodic boundary conditions, propagation characteristics are determined. The boundary condition is applied on the sides of the cell and a perfectly matched layer on the top and bottom open walls. Then, the phase constant between the two sides of the unit cell is fixed to 0 and the phase between other two sides of the unit cell is varied from 0 to $\pi$ degrees
Now, the propagation errors of the proposed regression model are compared with curve fitting model and experimental model. The propagation error deviation is determined in terms of percentage. The error deviations of curve fitting model and experimental model from proposed regression model are described in table I. The performance deviation of percentage of propagation deviation is illustrated in Fig.3. The formula is used for determining the percentage of error deviation is described as following them.

$\%$ of Error Deviation=[Proposed Model - Experimental(or Curve Fitting) Model] / Proposed Model

Table 1: Segmentation Points of Different PDGS and the Error Deviation.

\begin{tabular}{|l|l|l|}
\hline Propagation Characteristics & Dispersion & Transmission \\
\hline $\begin{array}{l}\text { Segmentation points of } \\
\text { Experimental Model }\end{array}$ & 45171.3 & 31465.9 \\
\hline $\begin{array}{l}\text { Segmentation points of } \\
\text { Curve Fitting Model }\end{array}$ & 45586 & 31633 \\
\hline $\begin{array}{l}\text { Segmentation points of } \\
\text { regression model }\end{array}$ & 45462.435 & 31586 \\
\hline $\begin{array}{l}\text { \% of Error Deviation from } \\
\text { experimental model }\end{array}$ & 0.6403 & 0.3801 \\
\hline $\begin{array}{l}\text { of Error Deviation from } \\
\text { curve fitting model }\end{array}$ & -0.2717 & -0.1488 \\
\hline
\end{tabular}

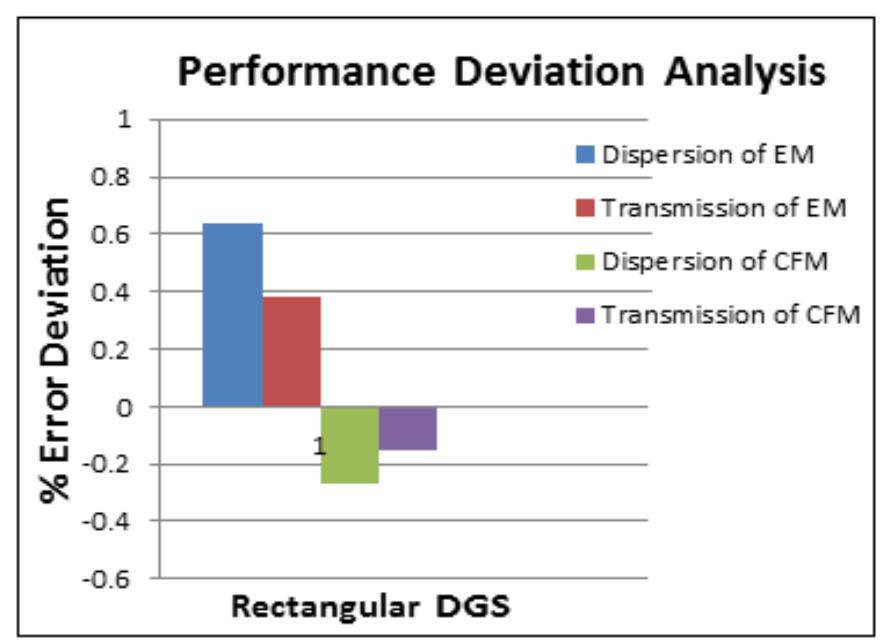

Fig 3: Performance Error Deviation Analysis of proposed model

In Fig. 3, the dispersion and surface transmission characteristics errors of periodic structures is analyzed for regression model, curve fitting model and experimental model. Then, the percentage of error deviation of the proposed model is determined from the experimental model and curve fitting model. The error deviation of rectangular periodic structure is 
plotted in bar chart. From the performance of error deviation, it is clear that the proposed regression analysis based mathematical model error is reduced remarkably. So, the proposed regression model based periodic structure characteristics analysis is better than the experimental model and curve fitting model. Hence, the proposed model characteristics analysis helps to design an accurate periodic structure and also reduces the propagation uncertainty

\section{CONCLUSIONS}

In this paper, regression analysis based mathematical model is proposed for evaluating the propagation uncertainty/error of DGS structures in microwave antenna. The proposed regression model is developed by using experimental data using regression analysis. Here, the considered DGS structures are rectangular shaped DGS structures. Dispersion and surface wave transmission coefficients are used to determine the propagation uncertainty. The bounded, leaky and brillouin wave zone boundary were chosen for analyzing the dispersion wave attenuation. The effectiveness of the proposed regression model is validated by comparing the error deviation of proposed model with curve fitting model and experimental model. By analyzing the error deviation performance of the proposed regression model with curve fitting model and experimental model, it says that the proposed method is better than curve fitting model and has less deviation from experimental data. Hence, it is more efficient in determining the accurate size of DGS in microwave antenna design.

\section{REFERENCES}

[1]. J Chen, Z B Weng, Y C Jiao and F S Zhang, "Low pass Filter Design of Hilbert Curve Ring Defected Ground Structure", Progress in Electromagnetics Research, Vol.70, pp.269-280, 2007

[2]. R Sharma, T Chakravarty, S Bhooshan and A B Bhattacharyya, "Design of a Novel 3dB Microstrip Backward Wave Coupler Using Defected Ground Structure", Progress In Electromagnetics Research, Vol.65, pp.261-273, 2006

[3]. X Q Chen, R Li, S J Shi, Q Wang, L Xu and X W Shi, "A Novel Low Pass Filter Using Elliptic Shape Defected Ground Structure", Progress in Electromagnetics Research, Vol.9, pp.117-126, 2008

[4]. L H Weng, Y C Guo, X W Shi and X Q Chen, "An Overview on Defected Ground Structure", Progress in Electromagnetics Research, Vol.7, pp.173-189, 2008

[5]. S H Zainud-Deen, M E Badr, E El-Deen, K H Awadalla and H A Sharshar, "Microstrip Antenna With Defected Ground Plane Structure as a Sensor For Landmines Detection", Progress In Electromagnetics Research, Vol.4, No.4, pp.27-39, 2008

[6]. H Liu, Z Li and X Sun, "Compact Defected Ground Structure in Microstrip Technology", Electronics Letters, Vol.41, No.3, pp.132-134, 2005
[7]. H Oraizi and M S Esfahlan, "Miniaturization of Wilkinson Power Dividers by Using Defected Ground Structures, Vol.4, pp.113-120, 2008

[8]. X Q Chen, L H Weng, Y C Guo and X W Shi, "RF Circuit Design Integrated with Microstrip DGS", Progress In Electromagnetics Research, Vol.3, pp.141-152, 2008

[9]. M Naghshvarian Jahromi and M Tayarani, "Miniature Planar UWB Bandpass Filters with Circular Slots in Ground", Progress In Electromagnetics Research Letters, Vol.3, pp.8793, 2008

[10]. S A Hosseini, Z Atlasbaf and K Forooraghi, "Two New Loaded Compact Planar Ultra-Wideband Antennas Using Defected Ground Structures", Progress In Electromagnetics Research, Vol.2, pp.165-176, 2008

[11]. J P Geng, J J Li, R H Jin, S Ye, X L Liang and M Z Li, "The Development of Curved Microstrip Antenna with Defected Ground Structure", Progress In Electromagnetics Research, Vol.98, pp.53-73, 2009

[12]. F J Wang and J S Zhang, "Wide Band Cavity-Backed Patch Antenna for PCS/IMI2000/2.4GHZ WLAN", Progress In Electromagnetics Research, Vol.74, pp.39-46, 2007

[13]. Arya Ashwini K, M V Kartikeyan and A Patnaik, "Defected Ground Structure in the perspective of Microstrip Antennas: A Review", Journal of RF-Engineering and Telecommunications, Vol.64, No.5-6, pp.79-84, 2010

[14]. M F Karim, A-Q Liu, A Alphones, X J Zhang and A B $\mathrm{Yu}$, "CPW band-stop filter using unloaded and loaded EBG structures", IEE Proceedings on Microwaves, Antennas and Propagation, pp.434-440, 2005

[15]. Heeduck Chae, Jong-Sik Lim, Cheol-Sig Pyo, Soon-Ick Jeon, Ji-Hoon Bae and Sangwook Nam, "Analysis of Periodically Loaded Defected Ground Structure and Application to Leaky Wave Antennas", Asia-Pacific Microwave Conference, 2003

\section{BIOGRAPHIE}

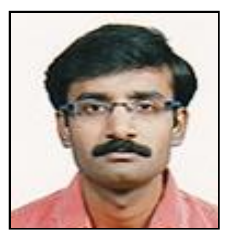

Prakash K Kuravatti, DOB: 18/09/1978, Place: Ranebennur, Dist.: Haveri, State: Karnataka. Working as an Assistant Professor, Alliance University, Bangalore, and have 7 years of Teaching Experience and papers are published in IJCA \& IJACR. 